\title{
The gastric cardia: what it reveals about our society
}

\author{
Martin Riegler
}

Published online: 6 November 2019

(C) Springer-Verlag GmbH Austria, part of Springer Nature 2019

Dear reader,

welcome to the last issue of European Surgery published in the 2010s of this millennium. Going in line with the recent issues, European Surgery serves the search towards the truth using the concepts, ideas and methods of accurate academical science. In addition, European Surgery aims to foster a mindset resisting the uncritical digestion of the so called leading and ruling opinion (the mental stool), which most frequently serves to increase and justify the power of multiple forms of interest (industries, politics). Take your time for these lines.

\section{Prologue}

According to this highly valuable purpose, this issue of European Surgery stages a spectrum of articles related to esophagology, endocrinology, gastrointestinal surgery and the peri-operative management around gut and bowel surgery. As thus the selection (logos!) and the composition (order! muse!) considers the very embryology of the human body: the thyroid, parathyroid glands and the thymus are the twins of the esophagus. During development these glands bulge out from the so called pharyngeal pouch. As a consequence, they share a common origin. In addition, the thyroids, parathyroid glands and the thymus are special manifestations of the so called submucosal glands of the esophagus. Histology finds that these glands share a common micro-architecture, which is based on the principle elements of the submucosal glands of the esophagus. Thus the thyroids, parathyroids and the thymus represent variations of of the motive

\section{Doz. Dr. M. Riegler $(\bowtie)$}

Reflux Medical, Mariannengasse 10/9, 1090 Wien, Austria martin.riegler@refluxmedical.com
(Bach, Beethoven, Brahms, Bruckner, Mahler) characteristic and typical for the development and construction of the esophageal submucosal glands. While the submucosal glands serve the production of mucus in the esophagus, those glands serve the production/ formatting of hormones and immune cells in other places (thyroid, parathyroid, thymus cell hormones and immune cell formatting within the epithelia lining those glands.). Most interesting, during disease, those twins of the esophagus start to remember their common embryological origin and form cells, glands and tissues which resemble well to less oriented submucosal glands of the esophagus (thyroiditis, parathyroid adenoma, thymus cell cancer, myasthenia etc.). Ask your pathologist, sit down with your pathologist and let him or her or her/him show you the histopathology of the esophagus, thyroid, parathyroid, thymus, using the microscopic examination using H\&E stained or immune stained slides of tissue coming from these organs. Thus you may enlarge your stage and attention for the large scale motive of the great image (Richard Wagner, Arnold Schönberg). As pointed out in one of the papers published in this issue of European Surgery. do not forget to accompany the examination of the slides with the administration of a good cup of Italian Naples-style espresso, because this method fosters a well-tuned and well-orchestrated bowel movement and gut function, even after abdominal surgery, as demonstrated within this issue of European Surgery.

Magnification matters, magnification counts, decides and opens your critical mind (I assume you have a critical mind), opens your awareness for details and definitely improves and enlarges your understanding of health and disease. We all are parts of the galaxy. We are really coming from Africa (200.000 years ago from South Africa, as published in the recent issue of Nature), therefore we are a family. Families love, hate, greed and behave like humans. Your calcium 
and sodium and phosphate share the same spacetime quanta, when compared to the ions in remote Hubble telescope envisioned galaxies. Therefore, dear reader, dear surgeon, dear physician, dear academic teacher, scholar, dean or researcher, mother and father, sister and brother, think about another impressive possibility, foster the use of a fundamental and unique opportunity, do not only talk about it, just go ahead and simply do it: allow yourself to go down to the level of histopathological magnification, which serves to define disease: i.e. histopathology, cellular pathology.

This complex and unique matter of fact exists, irrespective, if you are aware of it or not, if you think about it or not. The body does not care in as much you care about histopathology or not. Disease and health do not care, if you care about cellular pathology or not. Health and disease simply follow the principles of pathology. Nature tunes well, as long as we follow her path. Currently millions of young men and women are motivated to walk out of school and away from (their) nature. They are deceived by the dangerous mind set of green coloured hypocrites. However, envisioned from space, our globe appears definitely blue and white and brown, but not green at all. Maybe we should base our reasoning on a well-tested phenomenology (Prof. Edmund Husserl). Truth does not care about the way you might consider about the definition of health and disease, how you may think or suggest about the sources of disease. Who thinks? Who considers? Who out there finds time to think and consider more than superficial reasoning, reaction (twitter, facebook, google) and attention in 2019? If I were to look at the world today in 2019 I would definitely get the impression, that the large majority of humans stopped thinking, reasoning and so forth. Critical deep, open minded reasoning is replaced by acute reaction: copy, paste, open, close, store, delete. Remembrance is the past of our future. However, technologies have been developed, which should foster the acquisition of deep knowledge and ease any teaching and education. In contrast to that, stupidity seems to develop, exist and manifest almost everywhere. Stupidity seems to rule the globe. Wicked people botox their institutions, international mental spasms and achalasia regurgitate the tune: every plastic smile carries a stupid eye, yes we bolus, as such we can. Tears run down your cheeks to dry the lips which ceased to speak out wisdom fate for hunger. Stupidity seems to exists at a very large, immense, almost unimaginable scale, maybe comparable to the relevance and power of space time curving and spacetime quanta for the orientation of our universe. As thus our globe (world) continues to carry (tolerate) the burdens delivered by us (home base), invented by us (in field fly) and still carries us through the solar system (home run). Thank your globe for not giving up. Thank you readers for not giving up the critical appraisal. There has to be a chance for a change. And there is proof of this assumption. The proof is published within this issue of European Surgery, and we will come to that in a moment.

\section{Open your eyes}

Recognition equals the translation of cosmic fields of energies into treatable signs and perceptions. We continuously perceive. The sum of all our perceptions defines our momentary reality. The relevance of this reality for our momentary state of mood, temper and atmosphere, defines the truth. As such truth follows the principles of relativity, comes and goes, develops individually and models our momentary state of emotion, which in turn decides on our attention and selection of perceptions. As such closes the circuit of phenomenology. According to our experience, there is no perception without existence. As a consequence, perception models being and thus defines any moment of our existence. Based on this concept, thinking, feeling, sensing, reasoning, believing and stating these considerations models (is) the same: being (Parmenides, Heraclitus). The terms soul, mind, spirit, consciousness and atmosphere are linguistic means to indicate the same: the cosmic energy, i.e. fields of space time quanta (Rovelli $\mathrm{C}$ et al.), which translates into the form (particles, colours) of our momentary state of emotion (happiness, hope, excitement etc.): and this understanding defines (models) being.

You are allowed to perceive, but the source of energy, which orchestrates reasoning, observation, perception and consciousness cannot be thought, not even sensed and perceived. Whatever action a language may take, whatever large scale vocabulary you may invent: being itself cannot be perceived. Why that? The answer (model): "Because we assume, that being/truth is the state, which perceives and therefore cannot perceive and think itself (concept of truth)". Here language ceases to work. Beyond that remains pure emotion of the moment. We are only allowed to perceive manifestations of our being. No eye can watch itself. Therefore, we apply concepts to overcome this limitation of our being. Any concept equals a mirror, which pictures and models the eye (eye of Horus; the cosmic egg). Beyond that swallows the sea of emotions, the ocean of imaginations, the heaven of phantasies, the waves of music, thunder and emotions. Taken together, absolute truth is as it is, irrespective of the way, how we may or may not think, suggest and consider about it. Remains to improve our level of approximation towards the absolute truth. We are only allowed to reach out for approximations towards the truth. Please let us invent large scale mirrors for any form of magnification, ranging from the dimensions of particles to the far remote galaxies. Since the absolute truth equals thinking equals being we are limited within the context of our instrument: our mind. As such light serves to mirror space time curving. So let us proceed to approximate towards the absolute truth in surgical science. And go- 
ing in line with the actual issue of European Surgery, histopathology may in fact be one of the best ways to shed and approximate light, colours and recognition towards the dimensions of the so called absolute truth during clinical routine diagnosis and therapy. At this actual stage our minds reflux back to the globe: "Earth control to major Tom" (David Bowie).

The authors of the papers within this issue of $\mathrm{Eu}$ ropean Surgery are to be congratulated, their contributions are important and add to complete, increase and round up our understanding of surgical academical science and knowledge. Millions of times the earth danced around the sun, and it took those millions of times to foster the development of a human being, which in turn raised to question the cosmic clock phenomena and to invent science to explain how seasons, days, space time, spacetime quanta, cells and ions and molecules and energetic manifestations translate into our perceptions. The reviewers of the papers are to be thanked for their passion to go through those works and days (Hesiod) and joys and days (Proust) and to critically analyse the background, methods, results, conclusions, discussions, references, tables, figures and outlooks. Taken together, the authors, reviewers, editors, publisher (Springer) and you, dear reader, are to be thanked to support the major and fundamental and crucial aim of the project termed European Surgery: the will to foster the search for the truth, using the means, methods and techniques, technologies, innovations and conduct of reasoning of academical science related to surgery and beyond.

In the recent issues of European Surgery we examined the impact, role and importance of the so called ruling opinion for the revelation of the truth. We assessed in as much the ruling opinion may in fact inhibit the process of finding the truth. Going in line with our results we found, that, in the majority of the cases, the leading opinion rules, because it justifies and supports the interests of those in power. As such the ruling opinion serves the increase of power of specific inventions of human civilisation including institutions, politics, industries, religions and science. As thus academical studies may in fact become instruments to manipulate people in order to increase the power and influence of those standing behind these actions and activities. Generally spoken we should not negatively think and reason about this matter of fact. In the recent issue of European Surgery we clearly figured out the reason for these achievements and mechanisms. We assessed the so called "archeion", a network of nerve cells within our brain, which came up during the early development of the human brain. The "archeion" derived behaviour serves the mechanisms to assure survival of the human being against natural environmental enemies (other humans, animals, landscape, climate, asteroid impacts etc.). Taken together, the leading opinion is a natural matter of fact, it belongs to the nature of man and serves the will to power. As such we do not simply criticise the will to power. Without the will to power we would not have invented modern medicine, robot surgery, science, astronomy, physics, quantum state matters and arts. However, fire should be controlled to prevent that the human being gets burnt. Catch a fire. Ethiopia, the burnt faces, received the Nobel prize in 2019, Peter Handke got the Nobel prize in 2019. Going in line with the late Sir Karl Popper, he also contributed to question the ruling opinion and open our sensations for the hidden, the a-peiron, a term coined by the ancient thinker Anaximander, who has been brought back to our time by the physicist Prof. Carlo Rovelli and others. In addition, the hidden models the (absolute) truth and science aims to unhide the hidden and to reveal the truth for the benefit of man and nature. As thus we may return to live as harmonic members of the cosmic order and union, as least in the terms of a theory and a concept, that has been presented by Hesiod: those who lack the will to search for the truth will have to suffer from pain and oblivion. As such oblivion has been recognised as the common source of any disease. True? Yes! True!

\section{Critical science}

This issue of European Surgery contains an outstanding, fundamental and most important proof of the concept of Hesiod, the concept that drove the literature of amazing personalities including Friedrich Hölderlin, Robert Musil, Thomas Bernhard, Peter Handke, Thomas Mann, Stefan Zweig, Walt Whitman, Marcel Proust, James Joyce and many others. Those outstanding minds share the will to approximate the absolute truth, as much and as often and as much as possible. In addition they try to motivate us to follow their path and to resist the aggressive powers of any form of despotism, brain wash and mental slavery. Dear reader, at this moment, as you read those lines, ask yourself: whom do you serve, which form of despotism do you serve: facebook, twitter, google, PUB MED, medical industry, pharmaceutical industry, any politics, the cholesterol power game, the believe into poisoned pills stuck within the gastric cardia?

As general surgeons you may frequently be allowed to perform a so called esophago-gastro-duodenoscopy (EGD) (Figs. 1 and 2). Today this test is most frequently applied to assess the endoscopically visible signs of gastroesophageal reflux disease (GERD). GERD represents as a continuously rising life style disease and affects the life quality and the well being due to the symptoms and increases the cancer risk in those positive for Barrett's esophagus (BE) (Figs. 1 and 2). Why? Via dysplasia BE may progress to esophageal cancer in GERD symptom positive and negative individuals.

The ruling and leading opinion (RLO) teaches, that, at the level of the diaphragm, the lower end of the 


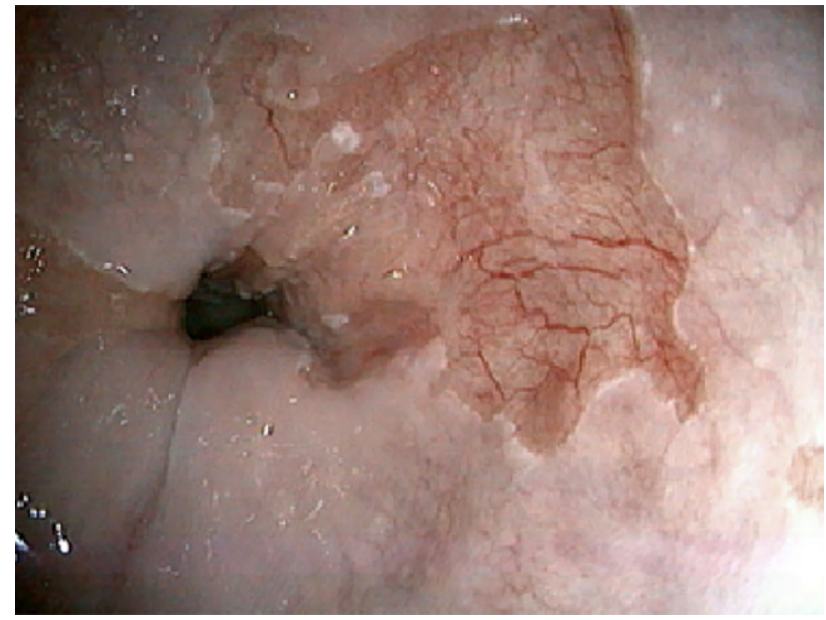

Fig. 1 Antegrade endoscopic image towards the distal esophagus at the level of the diaphragmatic impression. Note the presence of salmon coloured island of columnar lined esophagus, which develops as a consequence of gastroesophageal reflux. The histopathology of biopsies obtained from columnar lined esophagus may reveal presence or absence of Barrett's esophagus, as outlined in the text and in the paper by Chandrasoma published in this issue of European Surgery

normal, "healthy" and "happy" esophagus (i.e. the tube-shaped transporter of the food) fuses with the the proximal segment of the stomach, i.e. the gastric cardia. Thus, going in line with the ruling and leading opinion (RLO), the cardia represents the most proximal segment of the stomach, then follows the body and the antrum and the pylorus of the stomach. Accordingly, diseases of the gastric cardia where attributed to the stomach and treated as diseases of the stomach. For example, a cancer (adenocarcinoma) of the so called gastric cardia has been treated by resection of the stomach. PUB MED lists almost 9000 citations related to the gastric cardia. In addition, PUB med lists almost 5000 contributions related to the diagnosis and treatment of gastric cardia cancer (adenocarcinoma). This may involve at least more than 50,000 patients.

For more than 30 years the US expert pathologist Prof. Para Chandrasoma publishes data which convincingly demonstrate that the leading opinion (RLO) regarding the allocation of the cardia (stomach vs. esophagus) may be false. Going in line with his large body of published papers, books, reviews and editorials, what has been taken for gastric cardia in fact represents GERD damaged and injured lower end of the esophagus (Figs. 1 and 2). Thus, it seems, that, instead of the esophagus, the stomach has been removed for the treatment of a cancer of the esophagus in many thousand patients all over the globe, except for the institution, where Prof Chandrasoma works, i.e. the group around the US surgeon Prof Tom DeMeester.

In addition, Prof Chandrasoma developed an histopathology-based concept which allows the exact assessment of the cause of the disease: the impair-

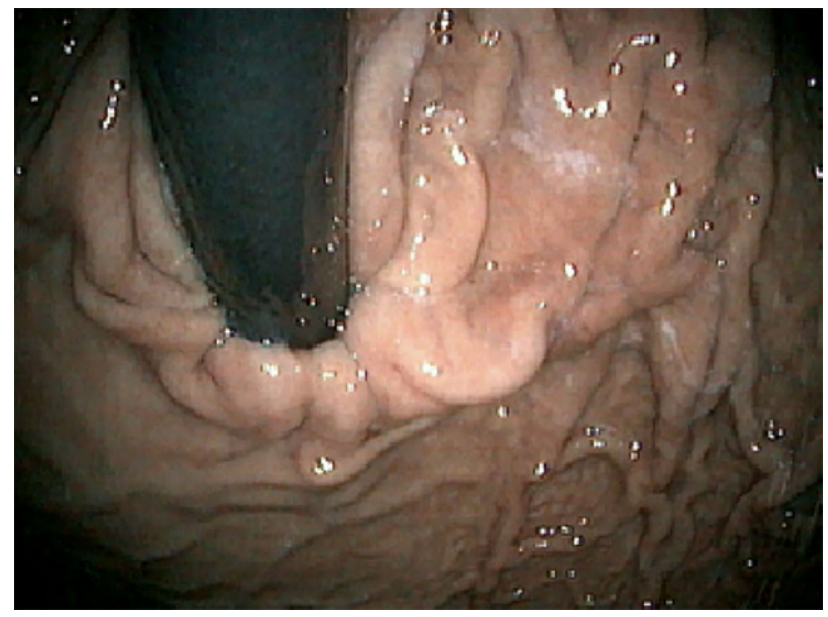

Fig. 2 Retrograde endoscopic image towards the distal esophagus. The histopathology of biopsies obtained from the mucosal folds surrounding the endoscope reveals, that was has been taken for gastric cardia in the past, in fact represents reflux injured columnar lined esophagus, as outlined in the text and in the paper by Chandrasoma published in this issue of European Surgery

ment of the function of the lower oesophageal sphincter. Recently the highly reputed and well known, famous US gastroenterologist Prof Stuart Spechler opened the awareness for the Chandrasoma concept. Within a review on GERD and BE, published in the recent issue of Gastroenterology, he confirmed the relevance and actuality of the Chandrasoma concept. He concluded, that we should alter our understanding of the so called gastric cardia. Going in line with this understanding European Surgery has published multiple papers and editorials in support of the Chandrasoma concept since more than 14 years. Again this proves, that European Surgery critically questions any leading and ruling opinion in medical science. At the end the story demonstrates, that our approach has not been a mistake. However, it took some time and required a lot of responsibility, passion and endurance. Finally truth withstood the resistance it coined and generated.

Therefore it is a great honour for European Surgery to be one of the first Journals in the world to be allowed to publish a contribution of Prof Para Chandrasoma after his concept has been officially accepted in the medical academical community. The Chandrasoma concept withstood the test of time. Thus the Chandrasoma paper within this issue of the journal may also contribute to foster your new understanding regarding the mechanisms and pathologies underlying the development of GERD and BE. May this also help you to improve treatment and cancer prevention.

Most importantly the Chandrasoma story proves, that in the large majority of cases, the leading opinion (RLO) serves the will to power, to hide the truth and to increase influence, power and might of those be- 
hind the scene. The ruling and leading opinion works, because the large majority of humans benefits from following a well accepted conduct of reasoning, when compared to create new concepts and ideas. Therefore dear reader, irrespective which role you play, take care: critically resist to be misused by powers and influences of any form of despotism. Question your daily routine. Look out to assess, recognise and detect any form of despotism, which aims to misuse your will and action. Take care not to be a victim of any form of despotism (industry, politics, academical societies). Otherwise you will have to pay the prize for your superficial reasoning and reaction. At this point I have to thank Prof Para Chandrasoma for his passion for not giving up to to unhide the truth, to reveal the truth, to give us the a-letheia, i.e. the unhidden, that has to be taken out of the river of oblivion (lethe). Therefore, the Chandrasoma story proves the actuality, relevance and importance of the concepts of Anaximander, Parmenides, Heraclitus, Husserl (phenomenology) and Eco (semiotics): we should never give up our search which aims to maximally approximate the absolute truth. As mentioned above we can not directly envision the absolute (ab-solvere) truth, because the instrument which aims to reveal the truth can not assess itself, the mind can not asses the mind, the spirit can not asses the spirit and soul can not assess the soul, the eyes can not assess themselves, we only can mirror the ab-solute truth (a-letheia) within the concepts, models and other forms of approximations, such as our actual, momentary state of emotion, mood, temper and atmosphere. Nothing else describes being. Maybe there may exist one fundamentally enlightened, crucial moment during our limited existence, when we may in fact be allowed to envision and sense the very and absolute truth (a-letheia), before we fuse with oblivion (lethe). Going in line with Thomas Bernhard, Correction "The end is no process. Clearing."

And there is an other aspect related to the gastric cardia. Going in line with the Chandrasoma concept, what has been taken for gastric cardia now represents reflux damaged esophagus. As thus the novel understanding of the "gastric cardia" reveals an underlying aspect of our society, which fosters the development of reflux and Barrett's oesophagus. Reflux is caused by an unhealthy life style including the continuous consumption of food and beverage containing concentrated sugar and artificial sugars. Essentially this points to an important quality of our modern society: we lack adequate discipline to resist the despotism of the food industry. We lack adequate discipline to fol- low an healthy life style. We lack adequate attention, respect and understanding for ourselves. Therefore, in addition to the above mentioned aspects related to science and the search for truth, the topic around the gastric cardia reminds us, that we would benefit from increased discipline and that we should stay away from an unhealthy life style and behaviour.

\section{Conclusion}

Taken together, this issue of European Surgery may foster an open minded approach against the uncritical digestion of the so called leading and ruling and misusing, despotic opinion for the benefit of the only and unique driving force of your academical science: the will for absolute truth. The papers should motivate you not to give up to resists against uncritical reasoning. Allow yourself deep reasoning, fundamental thoughts and take your time to place yourself within the waves of the universe. Finally those in the central European surgical societies who are members supporting the uncritical conduct of reasoning fostering the despotism of the ruling opinion may now be stimulated to remember the concept of truth, a-letheia. Stay tuned, enjoy the upcoming festivities and may health, happiness, satisfaction tune a positive vibration for you, for your families, friends and colleagues (those who are collecting the truth with and not AGAINST you!), stop being hypocrites (Bob Marley, No woman no cry: "observing the hypocrites, as they mingle with the good people we meet, good friends we had, good friends we have lost, along the way, in this great future, you can't forget your past, so dry your tears I say"), be true, be you, emancipate yourself from mental despotism and slavery,

\section{sincerely, Martin Riegler.}

Acknowledgements The author thanks his wife, parents, sister, family, friends and teachers, who support his scientific activities. He also thanks Springer and the team of editors, reviewers, and all the authors, who enable the realisation of the European Surgery project. Finally the author thanks the readers and hopes that some of the ideas may translate into an improvement of the reader's life quality.

Conflict of interest M. Riegler declares that he/she has no competing interests.

Publisher's Note Springer Nature remains neutral with regard to jurisdictional claims in published maps and institutional affiliations. 